\title{
On Shape Transformations and Shape Fluctuations of Cellular Compartments and Vesicles
}

\author{
E. Sackmann, J. Käs and J. Rädler \\ Physik Department (Biophysics Group), Technische Universität München, D-8046 Garching/München, Germany
}

Received April 1, 1993; accepted in revised form June 7, 1993

\begin{abstract}
We discuss the shape formation and shape transitions of simple bilayer vesicles in context with their role in biology. In the first part several classes of shape changes of vesicles of one lipid component are described and it is shown that these can be explained in terms of the bending energy concept in particular augmented by the bilayer coupling hypothesis. In the second part shape changes and vesicle fission of vesicles composed of membranes of lipid mixtures are reported. These are explained in terms of coupling between local curvature and phase separation.
\end{abstract}

\section{Introduction}

Lipid vesicles together with microemulsions form a new class of soft material (complex fluid) which fascinate by their outstanding elastic properties. These are not determined by surface tension as for liquid droplets but by bending and shear elasticity. Whereas the great present interest in microemulsions is stimulated by their technical applications, vesicular systems play a dominant role in living materials. In fact, the evolution of higher organism (e.g. cells) became only possible after nature found the trick with the (notably asymmetric) lipid bilayer $[1,2]$. As illustrated in Fig. 1 it enabled

- the creation of order by formation of functional compartments such as the mitochondria (as sites of the energy production) or the endoplasmatic reticulum (as the location of biosynthesis of proteins and lipids)

- to separate the space into sub-spaces of completely different composition

- the formation of shells of outstanding softness and stability against disrupture.

Since the lipid bilayers are excellent solvents for hydrophobic, semipolar and amphiphilic molecules they can accommodate large amounts of proteins (penetrating into or adsorbing to the bilayer). The lipid/protein bilayer has thus become the location of a large fraction of the biochemical reactions in cells. Their low dimensionality makes enzymatic reactions highly effective (cf. [2] for a review of physics and self organization of membranes, and [1] for the architecture and function of biomembranes).

Last but not least, lipid vesicles can undergo a large variety of local and global shape transformations involving an essential evolutionary advantage: namely the material and information exchange between the compartments of the cells and the cell interior and the hostile environment, respectively, by the budding-fission-fusion chain of events illustrated in Fig. 1.

It is this latter aspect of the extremely complex field of biomembrane physics which will be addressed in this paper. I will show that many of the cellular shapes or shape trans-

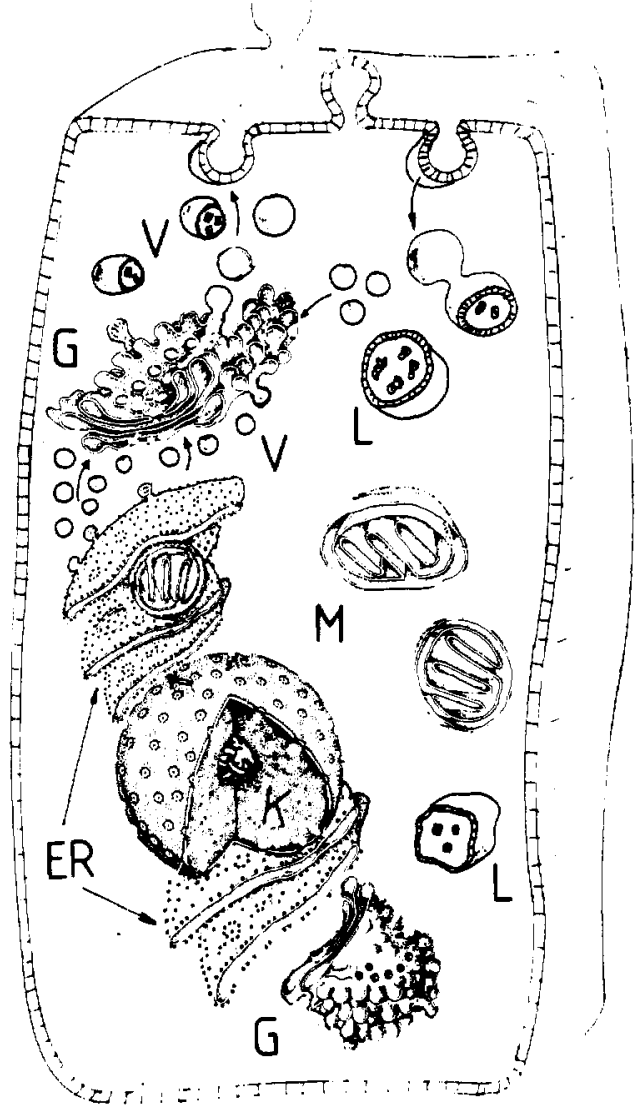

Fig. 1. Cartoon of cell stressing firstly, the modular design from functional compartments and secondly, the communication and material trafficking by vesicles. The major compartments are: (1) the nucleus, $K$, (the site of information storage); (2) the endoplasmatic reticulum, ER, with associated ribosomes (the location of biosynthesis as well as membrane assembly); (3) the Golgi apparatus, $\mathrm{G}$, (serving the modification and sorting of newly synthesized proteins and lipids and their directed distribution to other compartments and membranes); (4) the mitochrondria, M, (organelles where ATP is produced); (5) the lysosomes, $L$, (which serve the intracellular digestion and degradation of nutrition imported into the cell). $V$ denotes a whole palette of vesicles which serve the molecular transport between the cellular organelles and between the cell and its environment. The plasma membrane forms simultaneously a filter for selective molecular transport and serves the communication between cells.

The intracellular compartments are embedded in a soft network of protein filaments, the so-called cytoskeleton (not shown) which helps to establish some order within the plasma membrane and, together with the plasma membrane, serves the mechanical stabilization of the cell.

Last but not least it should be noted that the membranes divide the space into three subspaces: (1) the interior of the compartments (called lumina); (2) the zone between the compartments (the cytosol); and (3) the extracellular space.

Note that the vesicular transport (e.g. between ER and Golgi) ensures that the luminal spaces merge and that the inner and outer monolayers of the membranes are not interchanged. 
formations and instabilities can be mimicked by simple lipid bilayer vesicles composed of only one or two lipid components.

In the next section I will describe the shape transformations of one component membrane and show that complex membrane processes can be explained in terms of the simple bending energy concept augmented by the spontaneous curvature and monolayer coupling hypotheses.

In the final section shape transformations of mixed vesicles are described which are determined by coupling of local curvature and phase separation and it is shown that this is essential for fission processes.

\section{Biological shells}

Two typical examples of biological shell shapes are shown in Fig. 2: In Fig. 2(a) the evolution of a human erythrocyte is shown. It is born by budding from giant mother cells and rapidly expels its nucleus. The irregularly-shaped shell still contains some of the organelles, a high concentration of hemoglobin and two types of filamentous proteins: (1) spectrin, a highly flexible polypeptide of $100 \mathrm{~nm}$ contour length; and (2) actin oligomers. The latter are semiflexible filaments of variable length which are formed by linear condensation of actin monomers (polypeptides of $5.5 \mathrm{~nm}$ diameter). During a maturing process the latter proteins self-assemble into a quasi-two-dimensional network of triangular symmetry. The spectrin dimers form the sides and the actin oligomers (of $13 \mathrm{~nm}$ length) the corners. The network is loosely coupled to the lipid/protein bilayer by coupling proteins. As is also indicated in Fig. 2(b), decoupling of the network from the lipid protein bilayer in general leads to the blebbing of the lipid/protein bilayer. (For details of the structure see Refs $[2,4]$.)

The erythrocyte membrane is extremely soft with respect to shearing and bending, but laterally incompressible $[4,5]$. It is this unique combination of elastic properties which allows the cell to travel $400 \mathrm{~km}$ through very narrow capillaries during their 120-day lifetime. In fact, the bending modulus of the cell membrane is an order of magnitude smaller than that of a lipid bilayer of comparable composition [4]. Evidence has been provided that a large fraction of the energy consumption (in the form of ATP) of the cells is required to maintain this softness (the other part is required to maintain the ionic equilibrium). The mechanical properties of erythrocytes, their measurement and the structure of (a)
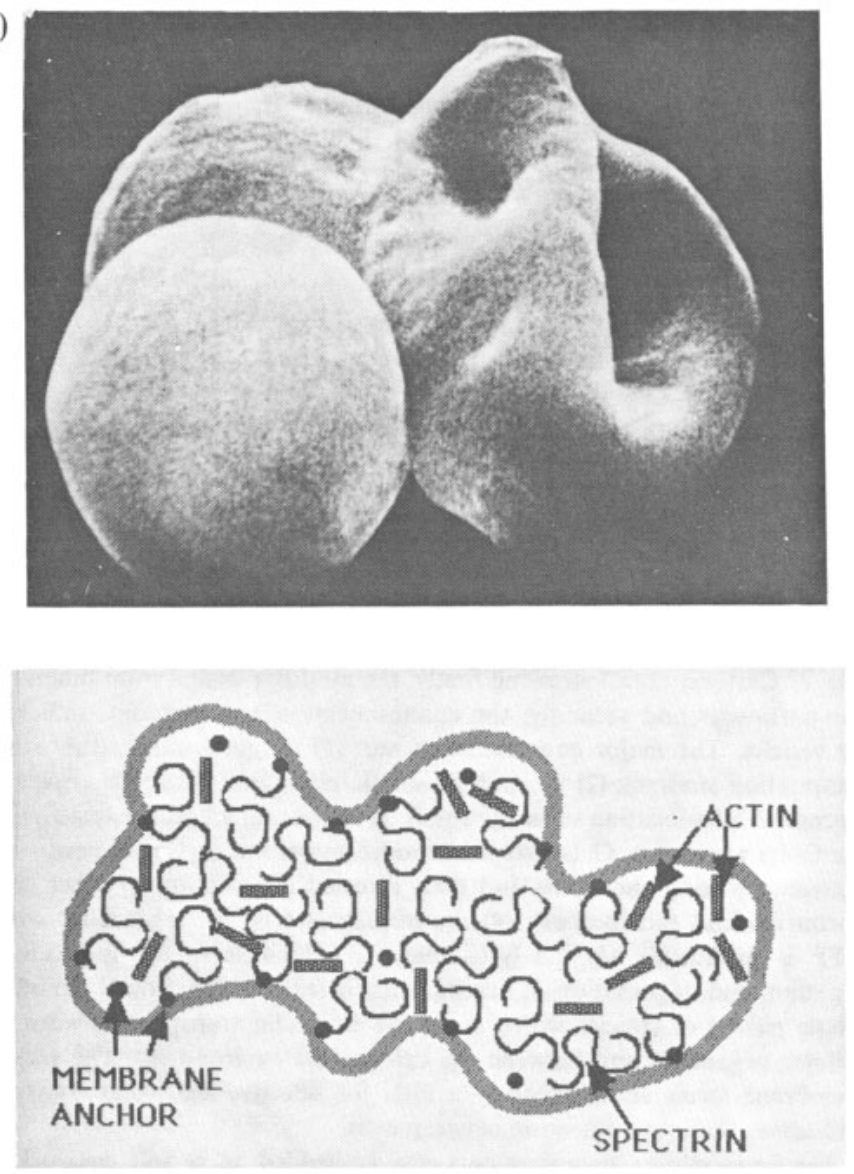
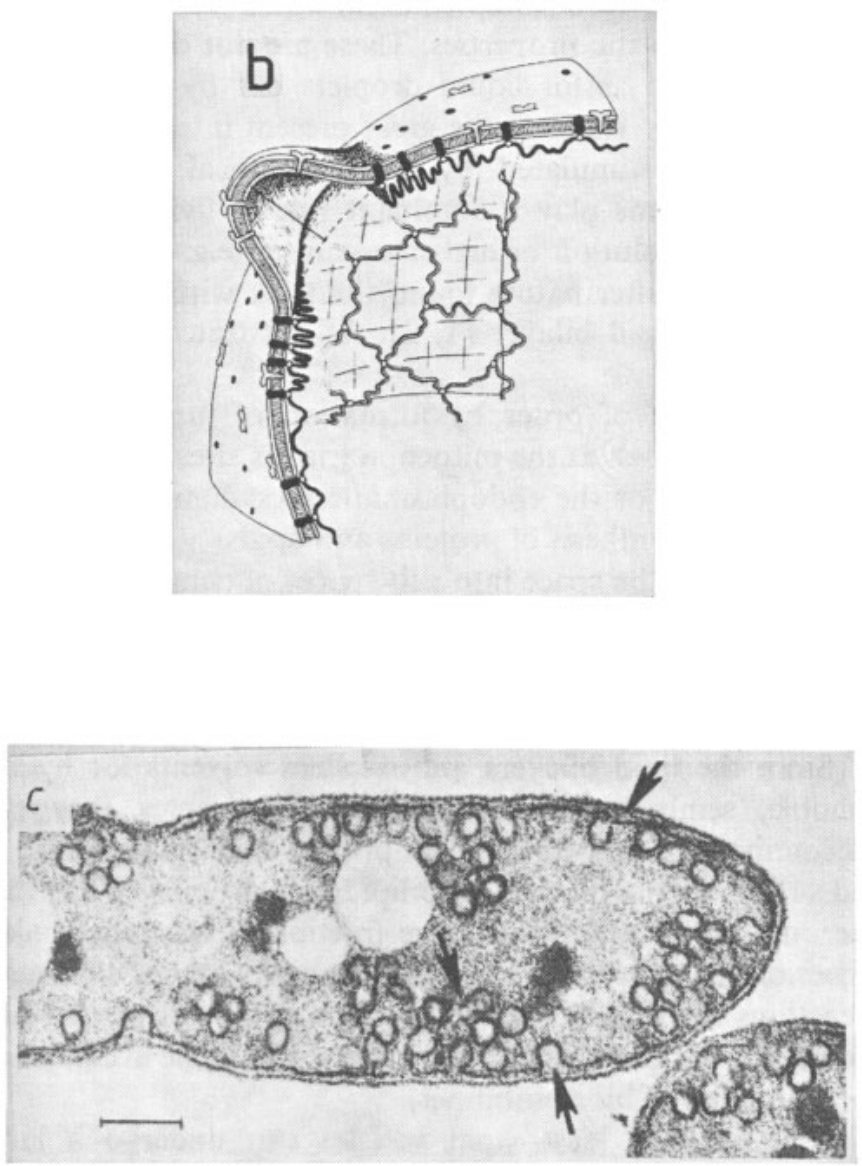

Fig. 2. (a) Shape transformation of erythrocyte during maturing, leading to highly symmetric discocyte shape. The cell is born by budding from a giant mother cell. It expells its nucleus as shown in the scanning electron micrograph. The highly irregular shape transforms eventually into a discocyte, a process which is closely associated with the formation of a quasi-two-dimensional macromolecular network beneath the inner leaflet of the plasma membrane (cf. Fig. 2(b) and Refs [2, 4]). (b) Illustration of stratified structure of plasma membrane which is made up from the liquid crystalline lipid protein bilayer (cf. [1]) and the membrane-coupled macromolecular networks. The latter is made up from highly flexible polypeptides of $L_{0} \sim 100 \mathrm{~nm}$ contour length (called spectrin) which is cross-linked by oligomers of actin (= rods of $\approx 13 \mathrm{~nm}$ length). The network forms a triangular lattice (with many disclinations) and is loosely coupled to the lipid protein bilayer via coupling proteins [4a]. The compound membrane is extremely soft with respect to bending and shearing but laterally incompressible [1]. (c) Electron micrograph of section through photosynthetic bacterium showing outer wall and inner membrane. The latter exhibits many tube-like and spherical invaginations the membrane of which carry the photosynthetic apparatus. 
the compound membrane are described elsewhere [5]. For a beautiful Monte Carlo simulation of the membrane elasticity see Boal et al. [6].

A completely different type of shell is shown in Fig. 2(c). The photosynthetic bacteria shown exhibits two outer membranes: the outer cell wall forming a protective shell and the inner membrane which contains the photosynthetic apparatus. The latter forms a network of tube-like and spherical invaginations. The space is again divided into three subspaces (the extracellular, the cytoplasmatic and that between the inner and outer membrane) which are preserved during the exchange of vesicles.

\section{Shape transitions of pure lipid bilayers by variation of excess area}

Vesicles composed of one component exhibit already a rich scenario of continuous and discontinuous shape changes by simply varying the temperature and therefore the area-tovolume ratio. The area and volume are measured from transverse sections by image processing taking advantage of the rotational symmetry $[7,8]$. Several characteristic examples are presented below:

(a) Sphere-discocyte-stomatocyte-budding transition. This transition (shown in Fig. 3) is typically observed if quasispherical (= tension free) veiscles are heated. These transform first into oblate shapes then into discocytes which eventually exhibit a symmetry breaking transition into the stomatocyte. The invagination of the latter becomes continuously (and reversibly) deeper upon further cooling until an irreversible transition into the inside budded state results. The latter transition occurs within a temperature interval of $0.1^{\circ} \mathrm{C}$ and is thus considered as discontinuous.

(b) Sphere-pear-outside budding transition. This shape change (shown in Fig. 4) is observed if one starts from a spherical vesicle which was kept under lateral stress for some time. It first becomes a prolate ellipsoid rather than a pear. While these changes occur continuously and reversibly, the transition to the outside budded shape (following after heating the pear) is discontinuous. Further increase of the area (by heating) results in the formation of a chain of small vesicles which exhibit a pronounced hysteresis after recooling.

(c) Blebbing transition. This transition shown in Fig. 5 is puzzling. It is observed by heating a vesicle which was also kept under tension (e.g. osmotically) for some time before heating. The vesicle assumes first a slightly edgy shape showing that excess area is generated. This is followed by a sudden formation of one or several protrusions which may be spherical or tube-like. They can point to the outside or inside. The excess area of the vesicle after blebbing is larger than estimated from the well-known area expansitivity. The reservoir for this excess area is still unknown since it appears that the vesicle was single-shelled.

(d) Osmotic driven shape changes. It is intuitively clear that all shape transitions can also be induced by osmotic effects or chemically-induced bending moments (e.g. mediated by macromolecular adsorption). An example of an osmotically driven sphere $\rightarrow$ stomatocyte transition is exhibited in Fig. 6.

Under certain conditions of preparation vesicles with toroidal shape were observed by Bensimon et al. [12].

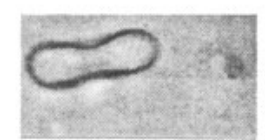

$\mathrm{T}=40.1^{\circ} \mathrm{C}$

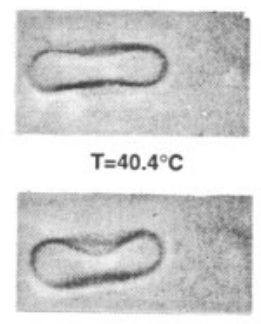

$\mathrm{T}=40.7^{\circ} \mathrm{C}$

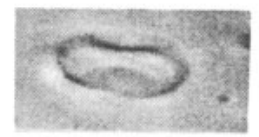

$\mathrm{T}=41.0^{\circ} \mathrm{C}$

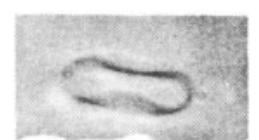

$\mathrm{T}=41.3^{\circ} \mathrm{C}$

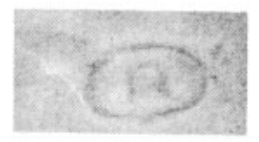

$\mathrm{T}=41.6^{\circ} \mathrm{C}$

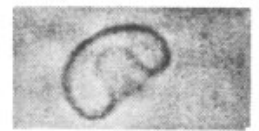

$\mathrm{T}=41.9^{\circ} \mathrm{C}$

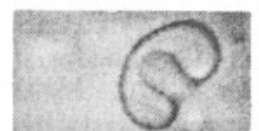

$\mathrm{T}=42.2^{\circ} \mathrm{C}$

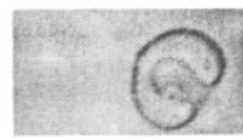

$3 \mathrm{sec} \quad \mathrm{T}=42.2^{\circ} \mathrm{C}$

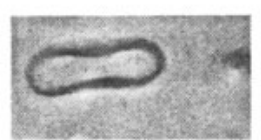

$\mathrm{T}=40.2^{\circ} \mathrm{C}$

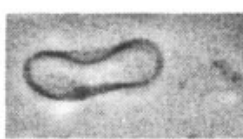

$\mathrm{T}=40.5^{\circ} \mathrm{C}$

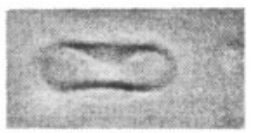

$\mathrm{T}=40.8^{\circ} \mathrm{C}$

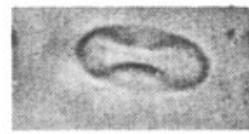

$\mathrm{T}=41.1^{\circ} \mathrm{C}$

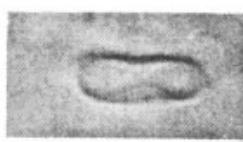

$\mathrm{T}=41.4^{\circ} \mathrm{C}$

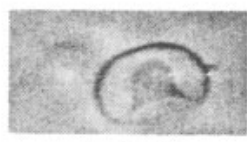

$\mathrm{T}=41.7^{\circ} \mathrm{C}$

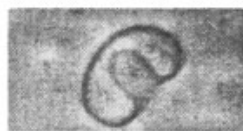

$\mathrm{T}=42.0^{\circ} \mathrm{C}$

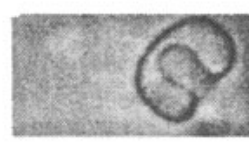

$1 \mathrm{sec} \quad T=42.2^{\circ} \mathrm{C}$

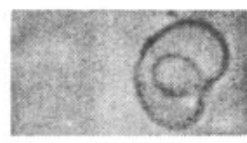

$4 \mathrm{sec} \quad \mathrm{T}=42.2^{\circ} \mathrm{C}$

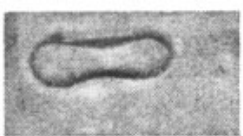

$\mathrm{T}=40.3^{\circ} \mathrm{C}$

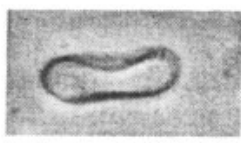

$\mathrm{T}=40.6^{\circ} \mathrm{C}$

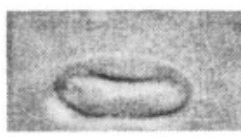

$\mathrm{T}=40.9^{\circ} \mathrm{C}$

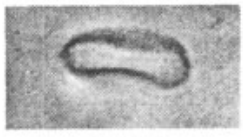

$\mathrm{T}=41.2^{\circ} \mathrm{C}$

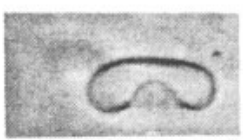

$\mathrm{T}=41.5^{\circ} \mathrm{C}$

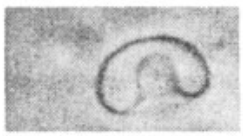

$\mathrm{T}=41.8^{\circ} \mathrm{C}$

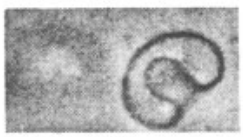

$\mathrm{T}=42.1^{\circ} \mathrm{C}$

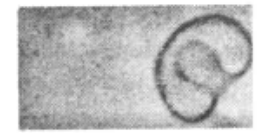

$2 \mathrm{sec} T=42.2^{\circ} \mathrm{C}$

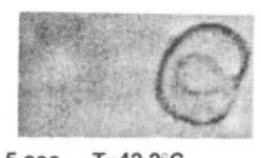

Fig. 3. Sequence of continuous and discontinuous shape change of DMPC vesicle driven by increase of excess-area to volume ratio. The initial state was quasi-spherical ( = tension free). The transition to the prolate ellipsoid over the discocyte to the stomatocyte is continuous. The final bud formation of the latter is discontinuous. Note the symmetry breaking of the discocyte which is indicated by arrow.

\subsection{Hysteresis of shape transitions}

These are most remarkable and poorly understood. One example is shown in Fig. 7. By cooling an outside-budded vesicle the area of the bud reduces in a cyclic way by repeated opening and closing of the neck connecting the bud with the mother vesicle. The last step consists in the transition to the pear.

Another pathway of the retraction of chains of vesicles is by quantized reduction of the number of vesicles which occurs without a remarkable change of the vesicle radii. Since this pathway is accompanied by a reduction in the vesicle volume [8] a hole is formed during each retraction step.

Two explanations for the hysteresis effects have recently been given: (1) van der Waals attraction between the mother cell and the bud and (2) a non-linear bilayer elasticity in the 


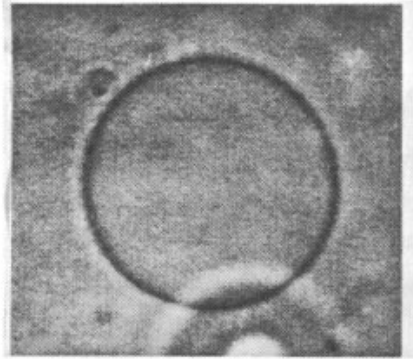

(1): $T=27.2^{\circ} \mathrm{C} \mathrm{V}=12200 \mu \mathrm{m}^{3} \mathrm{~A}=2570 \mu \mathrm{m}^{2}$

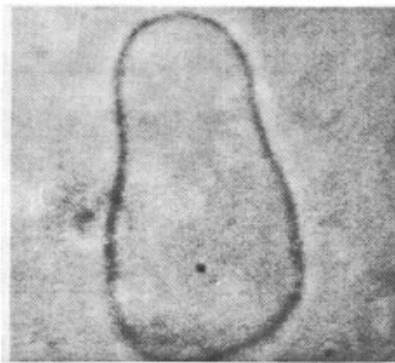

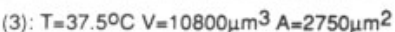

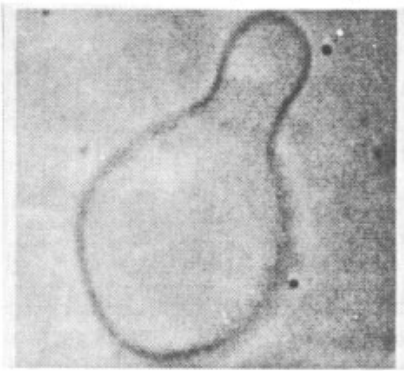

(5): $T=41.0^{\circ} \mathrm{C} \mathrm{V}=11900 \mu \mathrm{m}^{3} \mathrm{~A}=2810 \mu \mathrm{m}^{2}$

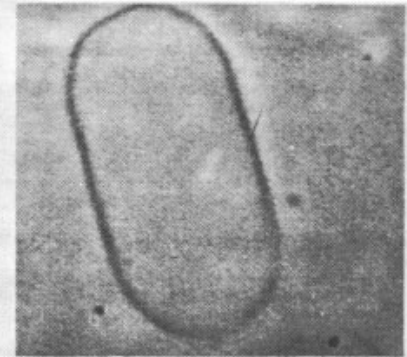

(2): $T=36.0^{\circ} \mathrm{C} V=12200 \mu m^{3} \mathrm{~A}=2770 \mu \mathrm{m}^{2}$

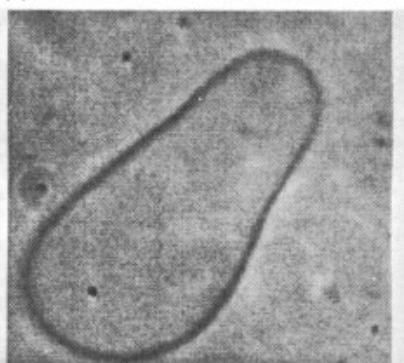

(4): $T=39.1^{\circ} \mathrm{C} \mathrm{V}=12000 \mu m^{3} \mathrm{~A}=2800 \mu \mathrm{m}^{2}$

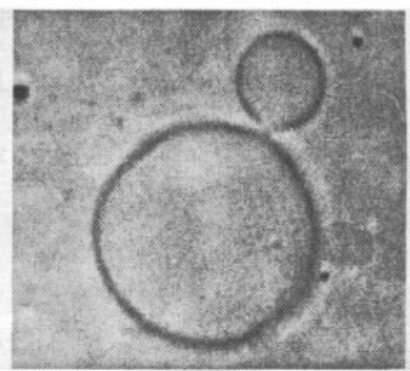

$10 \mu \mathrm{m}$

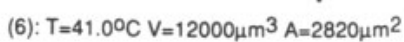

Fig. 4. Budding of vesicle via continuous prolate ellipsoid $\rightarrow$ pear transition. The transition from the pear to the budded shape (last image) occurs within a temperature interval of $0.1{ }^{\circ} \mathrm{C}$ (from 40.9 to $41^{\circ} \mathrm{C}$ ) and is thus considered as discontinuous. The initial state is spherical and under lateral stress. Upon heating above $41^{\circ} \mathrm{C}$ a chain of beads forms. The transition exhibits a pronounced hysteresis (cf. Fig. 7). (Source Ref. [7].)

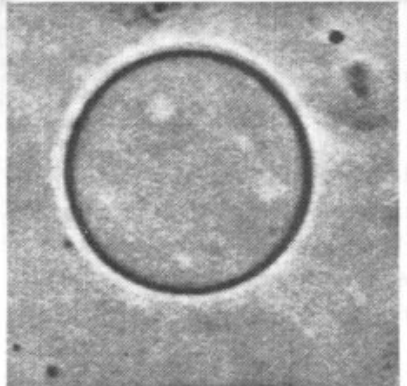

(1): $T=27.2^{\circ} \mathrm{C} V=11008 \mu \mathrm{m}^{3} \quad A=2401 \mu m^{2}$

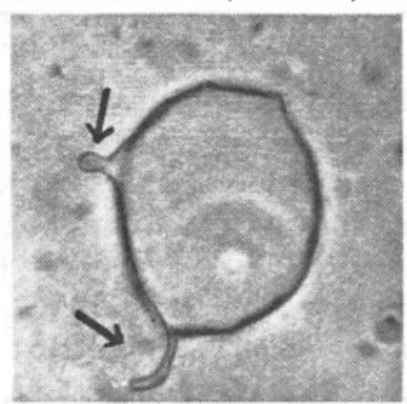

(3): $T=37.1^{\circ} \mathrm{C} V=11008 \mu m^{3} A=2568 \mu m^{2}$

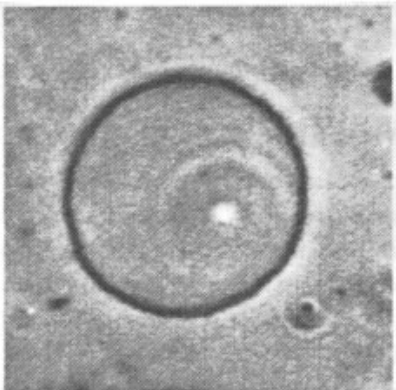

(2): $T=35.8^{\circ} \mathrm{C} \quad V=11008 \mu m^{3} \quad A=2546 \mu m^{2}$

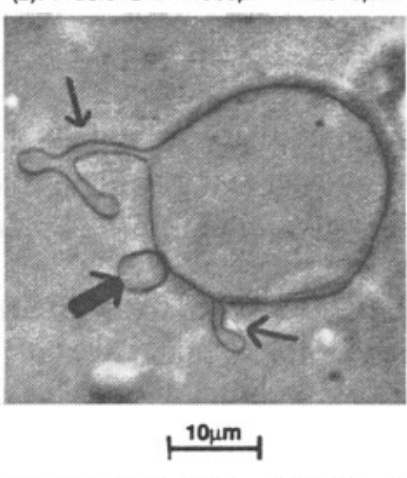

(4): $T=37.1^{\circ} \mathrm{C} \mathrm{V}=11008 \mu \mathrm{m}^{3} \mathrm{~A}=2575 \mu \mathrm{m}^{2}$
Fig. 5. Blebbing transition of DMPC vesicle in pure water by increase of the area by only about $7 \%$ (according to [8]). The areas given in the figure were only measured for the initial spherical shape while the values after blebbing were estimated by assuming an area expansivity of $6 \times 10^{-3} \mathrm{~K}^{-1}$.
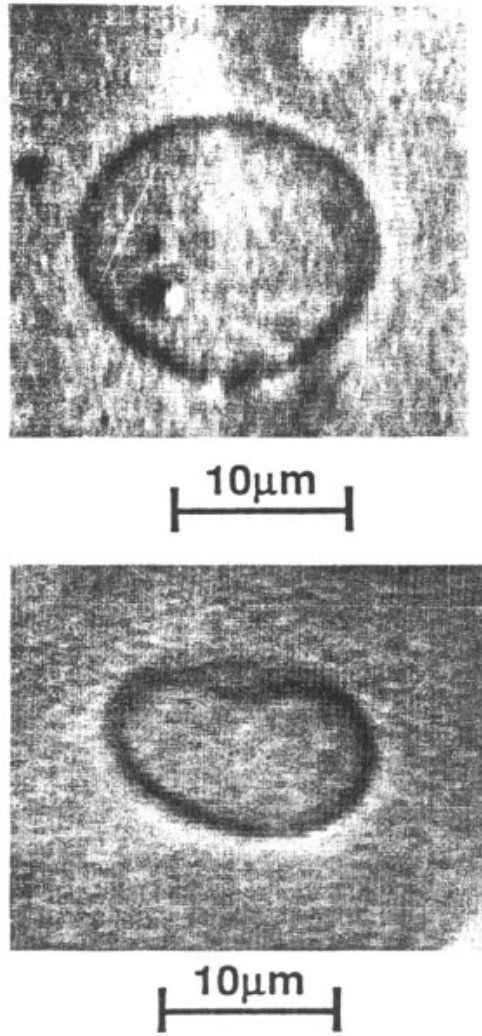

Fig. 6. Example of osmotically-induced shape change. DMPC was swollen in 200 mosm inositol. Then the inositol at the outside was continuously replaced by an iso-osmolar $(100 \mathrm{mM}) \mathrm{NaCl}$ solution via dialysis tubes. (a) Inside and outside containing inositol. (b) Shape observed after replacing the outside medium by $178 \mathrm{mM}$ inositol and $11 \mathrm{mM} \mathrm{NaCl}$. (Source Ref. [7].)

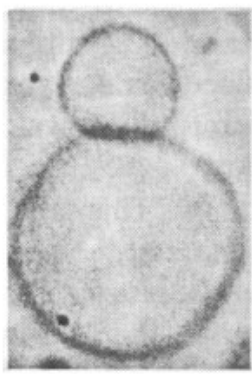

(1): $\mathrm{T}=52.6^{\circ} \mathrm{C}$

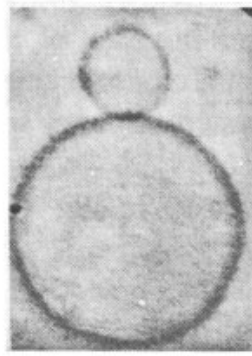

(4): $\mathrm{T}=43.6^{\circ} \mathrm{C}$

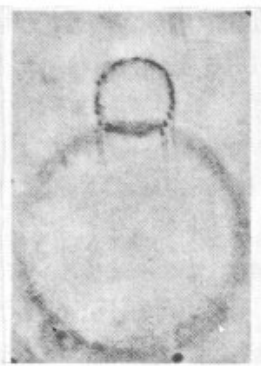

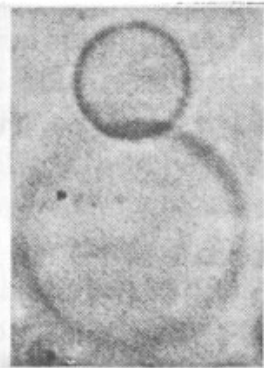

(2): $\mathrm{T}=50.8^{\circ} \mathrm{C}$

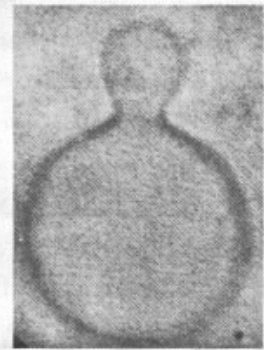

(5): $\mathrm{T}=41.4^{\circ} \mathrm{C}$

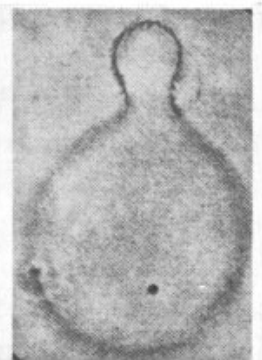

(8): $\mathrm{T}=31.70 \mathrm{C}$

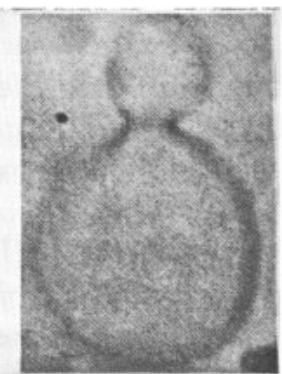

(3): $\mathrm{T}=49.6^{\circ} \mathrm{C}$

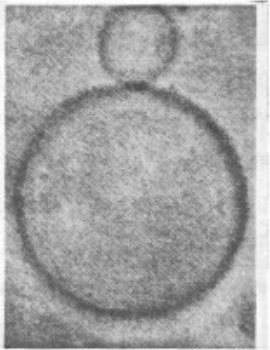

(6): $\mathrm{T}=39.6^{\circ} \mathrm{C}$

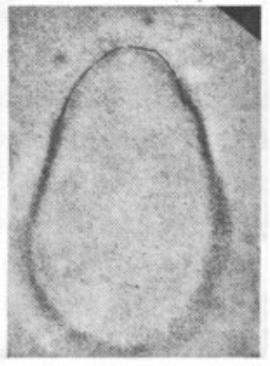

$10 \mu \mathrm{m}$
Fig. 7. Hysteresis of budding transition of DMPC vesicle. Reduction of the excess area by cooling leads to a cyclic decrease of the size of the bud which occurs by transient widening and re-closing of the neck. (Source Ref. [7].) 
bilayer coupling model [9]. It has already been shown by Bruinsma [10], that in the absence of salt, the van der Waals attraction is sufficient to induce tube formation (cf. also Bloom et al. 1991 [11]).

\subsection{The bending energy concept of vesicle shape changes}

The bending energy concept $[13,14]$ states that the vesicle shape is determined by the minimum of the bending energy

$G_{\text {bend }}=\frac{1}{2} K_{\mathrm{c}} \int \mathrm{d} A\left(C_{1}+C_{2}\right)^{2}$

where $C_{1}$ and $C_{2}$ are the principle radii of curvature. [Note that $C_{i}=\partial^{2} u(\boldsymbol{r}) / \partial x_{i}^{2}$ where $u(\boldsymbol{r})$ denotes the surface of the vesicle.] $K_{\mathrm{c}}$ is the bending moduius. The actual situation is, however, very complex and depends on the constraints and the symmetry of the membrane as follows $[8,13-15]$.

(1) In general the exchange of lipids between the membrane and the aqueous environment is very slow (order of hours). Moreover, the osmotic equilibrium is rapidly established. For that reason the average membrane area $\langle A\rangle$ and the volume $V$ are constant.

(2) If the inner and outer aqueous media are different one has to consider an intrinsic bending moment, $M$, resulting in a spontaneous curvature

$C_{0}=M / K_{\mathrm{c}}$

This can be considered by including a term $-C_{0}$ in the bracket of eq. (2) yielding the spontaneous curvature model of Helfrich [13].

(3) The lipid exchange between the two opposing monolayers is very slow (of the order of 10 hours) and for closed vesicles the resistance to bending is determined by the lateral expansion of the monolayer on the convex side and the lateral compression on the concave side of the bilayer [14]. This implies the additional constraint that the difference between the areas of the inner and outer monolayer is fixed. This condition can be expressed as [14]

$\Delta A=d_{m} \int \mathrm{d} A\left(C_{1}+C_{2}\right)$

where $d_{m}$ is the distance between the centers of the two monolayers. The integration has to be performed over the inner and the outer monolayer. Owing to this so-called bilayer-coupling constraint, vesicles behave quite differently from microemulsions. The membrane asymmetry can be accounted for by introduction of an intrinsic area difference $\Delta A_{0}[15]$.

As shown by Seifert et al. [17], the spontaneous curvature model [13] and the bilayer coupling model [14-16] can be combined in a general curvature energy functional

$F_{\text {ela }}=\frac{1}{2} K_{\mathrm{c}} \int \mathrm{d} A\left(C_{1}+C_{2}+C_{0}\right)^{2}+\frac{1}{2} K_{\mathrm{c}}^{\prime}\left(\Delta A-\Delta A_{0}\right)^{2}$

$K_{\mathrm{c}}$ and $K_{\mathrm{c}}^{\prime}$ are somewhat different bending elastic moduli. $K_{\mathrm{c}}$ is analogous to the splay elastic constant of liquid crystals $\left(K_{11}\right)$ and can be expressed as $K_{\mathrm{c}}=2 K_{11} * d_{m}$. It is determined by the mutual tilting of the lipid molecules during bending.

$K_{\mathrm{c}}^{\prime}$ is related to the lateral compressibilities of the outer $\left(K_{0}\right)$ and the inner monolayer $\left(K_{1}\right)$ as [14]

$K_{\mathrm{c}}^{\prime}=d_{\mathrm{m}}^{2} \frac{K_{0} K_{\mathrm{i}}}{K_{0}+K_{\mathrm{i}}}$

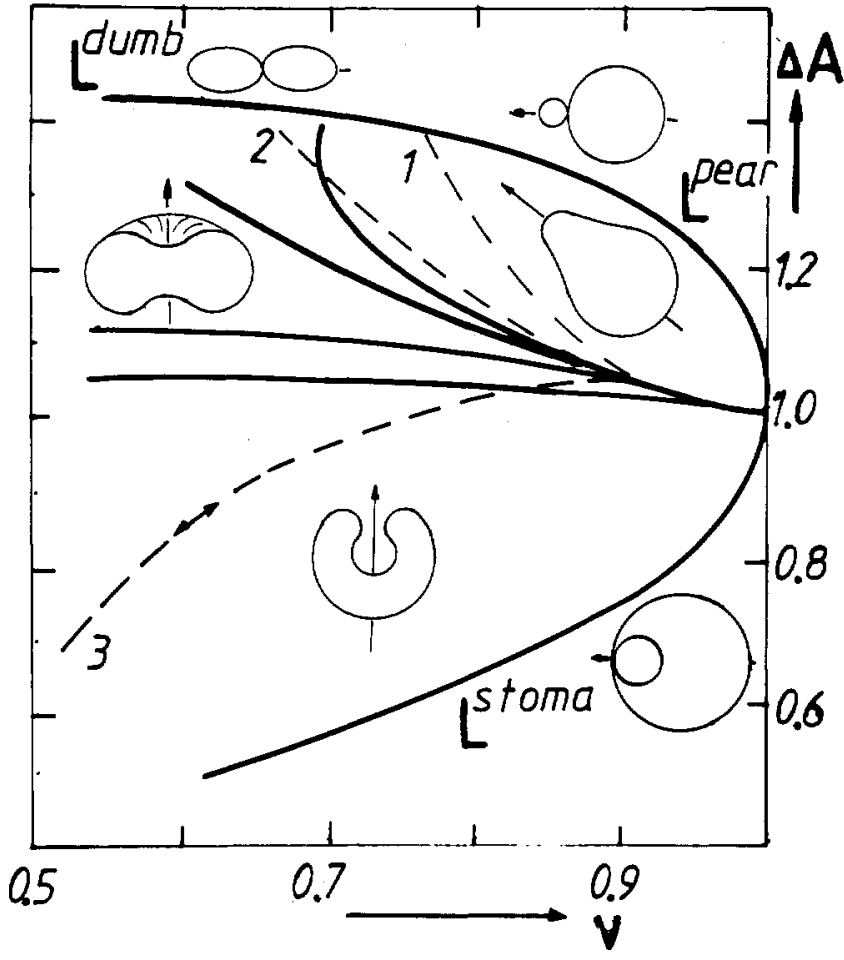

Fig. 8. Phase diagram of vesicle shapes as calculated by the bilayer coupling model. $\Delta a$ and $v$ are the reduced area difference and the reduced volume, respectively, defined in eqs (7). Phase boundaries $L_{i}$ define the limiting budded shapes. Note that $\Delta a>0$ corresponds to a positive and $\Delta a<0$ to a negative spontaneous curvature. The spherical shape corresponds to $\Delta a=v=1$.

The dashed curves 1, 2, 3 denote observed shape changes. Thus 1 denotes the sphere-pear-outside budded transition of Fig. 4. (Source Ref. [7].)

Since the splay elasticity is also determined by the variation of the lateral compressibilities across the bilayer [19], the two bending moduli are about equal. Since $K_{\mathrm{c}}^{\prime}$ is determined by the global constraint of fixed area $\langle A\rangle=$ const, it is also called the global bending modulus, while $K_{\mathrm{c}}$ is called the local modulus.

It is helpful to realize that in the classical theory of shells the bending rigidities would be related to the Young modulus, $E$, and the Poisson ratio $v$, by

$K_{\mathrm{c}}=E\left(2 d_{\mathrm{m}}\right)^{3} / 12\left(1-v^{2}\right)$

Both models are related by a Legendre transformation and yield therefore the same stable shapes [7]. However, the models predict different pathways of shape changes and different continuities. Thus the prolate ellipsoid-to-pear transition is predicted to be discontinuous in the $C_{0}$-model which disagrees with the observation [8]. In contrast, the bilayer coupling model predcits the right behaviour. Other examples were discussed previously [8].

The generalized model of eq. (5) comprises all situations. Depending on the ratio $K_{\mathrm{c}} / K_{\mathrm{c}}^{\prime}$ the $C_{0}$-model $\left(K_{\mathrm{c}} / K_{\mathrm{c}}^{\prime} \rightarrow \infty\right)$ or the bilayer coupling model $\left(K_{\mathrm{c}} / K_{\mathrm{c}}^{\prime} \rightarrow 0\right)$ dominates the vesicle shape. Seifert et al. [17] have calculated a complete phase diagram of vesicle shapes. Figure 8 shows calculated shapes of minimum bending energy in a $v-\Delta a$ phase diagram, where $v$ and $\Delta a$ are the reduced volume and area difference defined as

$v=\frac{V}{(4 \pi / 3)\left(A_{\mathrm{i}} / 4 \pi\right)^{3 / 2}}, \quad \Delta a=\frac{\Delta A}{\left(A_{\mathrm{i}} / 4 \pi\right)^{1 / 2} 8 \pi d_{\mathrm{m}}}$ 
where $A_{\mathrm{i}}$ is the area of the inner monolayer. The denominator is equal to the radius of a sphere with the same volume, $V$, a vesicle considered (and is therefore called equivalent radius) value of this phase diagram is that it allows to calculate path phase transitions by comparison of experimental and theoretical shapes. This is essential since the shape transitions depend critically on the pre-history. Thus, as shown in Figs 3-5 sphere vesicles kept under high lateral tension for an extended peripheral time (e.g. 1 hour) behave very different from flaccid vesicles. The reason is that in the former case lipids can exchange between two monolayers and the initial $\Delta A$-value for the two situations differ drastically [7].

\section{Shape changes of lipid alloys are determined by coupling of phase separation and curvature}

Shape changes of mixed lipid vesicles are much more complex for three reasons: (1) They are determined by the coupling of curvature and phase separation. (2) The lipids are in general asymmetrically distributed between the monolayers leading to spontaneous curvature effects. (3) Whereas fluid vesicles of pure lipids are very stable even after budding, vesicle fission is quite common for mixtures. In fact there are two limiting types of behaviour: domain formation and vesicle detachment.

The coupling between curvature and phase separation is obvious. In a fluid bilayer undergoing budding, lipids with large head groups will for instance accumulate in the outer monolayer of the bud. In the case of lateral phase separation into a fluid and a crystalline phase (e.g. during cooling) the crystalline lipid forms flat patches while the fluid component accumulates in the curved regions so that the vesicles assume a polygonal shape. Some examples are shown below. Many more have been reported previously (cf. [1]).

Figure 9 shows lateral phase separation induced by macromolecular adsorption which causes simultaneously lateral phase separation. In this case the vesicle consisted of a 1:1 mixture of a negatively charged and a non-charged lipid. Addition of poly-lysine (or other macromolecules with excess positive charges) bind electrostatically to the charged lipid causing lateral phase separation. Depending on the amount of charged lipid present, the vesicle can become unstable leading to the detachment of small vesicles or it assumes a domain structure as shown in Fig. 9. Similar effects are caused by the addition of divalent ions (e.g. $\mathrm{Ca}^{++}$). One outstanding property of such charge-induced phase separation effects is that they exhibit a strong hyster-

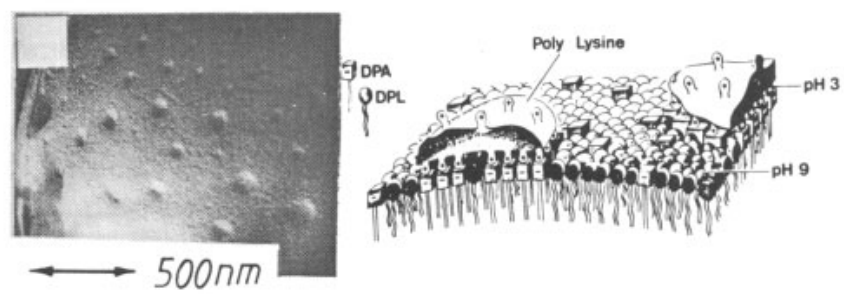

Fig. 9. Domain formation in mixed vesicles undergoing phase separation Examples of circular protrusions in mixture of charged and non-charged lipid by adsorption of macromolecules (here poly-lysine) exhibiting opposite charges as the lipid. Note the nearly hexagonal arrangement of the buds. This type of budding induced by macromolecular adsorption is reminiscent of coated pit formation in cells (cf. [1]). esis (cf. [1]). At high charge densities (>50 mol.\% of lipid) the vesicles become unstable and aggregate into multilamellar systems.

A completely different example of a shape change caused by phase separation into a fluid and a crystalline phase (or two crystalline phases) is shown in Fig. 10. The vesicle consists of a partially cross-linked (= tethered) membrane [12b, 18]. It is composed of a normal lecithin and polymerizable diacetylene lecithin. The latter can be photochemically cross-linked [top of Fig. 10(a)] and both lipids can unergo a chain melting transition. Depending on the concentration, the cross-linked diacetylene lipid forms gel-like patches which are embedded in a fluid matrix of DMPC or fluid DMPC domains are embedded in the gel resulting in a twodimensional sponge. In the fluid state the vesicle assumes a swollen quasi-spherical shape exhibiting a domain structure [18]. After cooling below the phase transition of one or both components, a wrinkling transition occurs and the vesicle assumes a crumbled shape. The transition is completely reversible. The same phenomenon is observed for vesicles of pure diacetylene by polymerizing only part of the lipid [12b]. Complete polymerization leads to the destablization of the vesicles which decay into multilamellar aggregates [18].
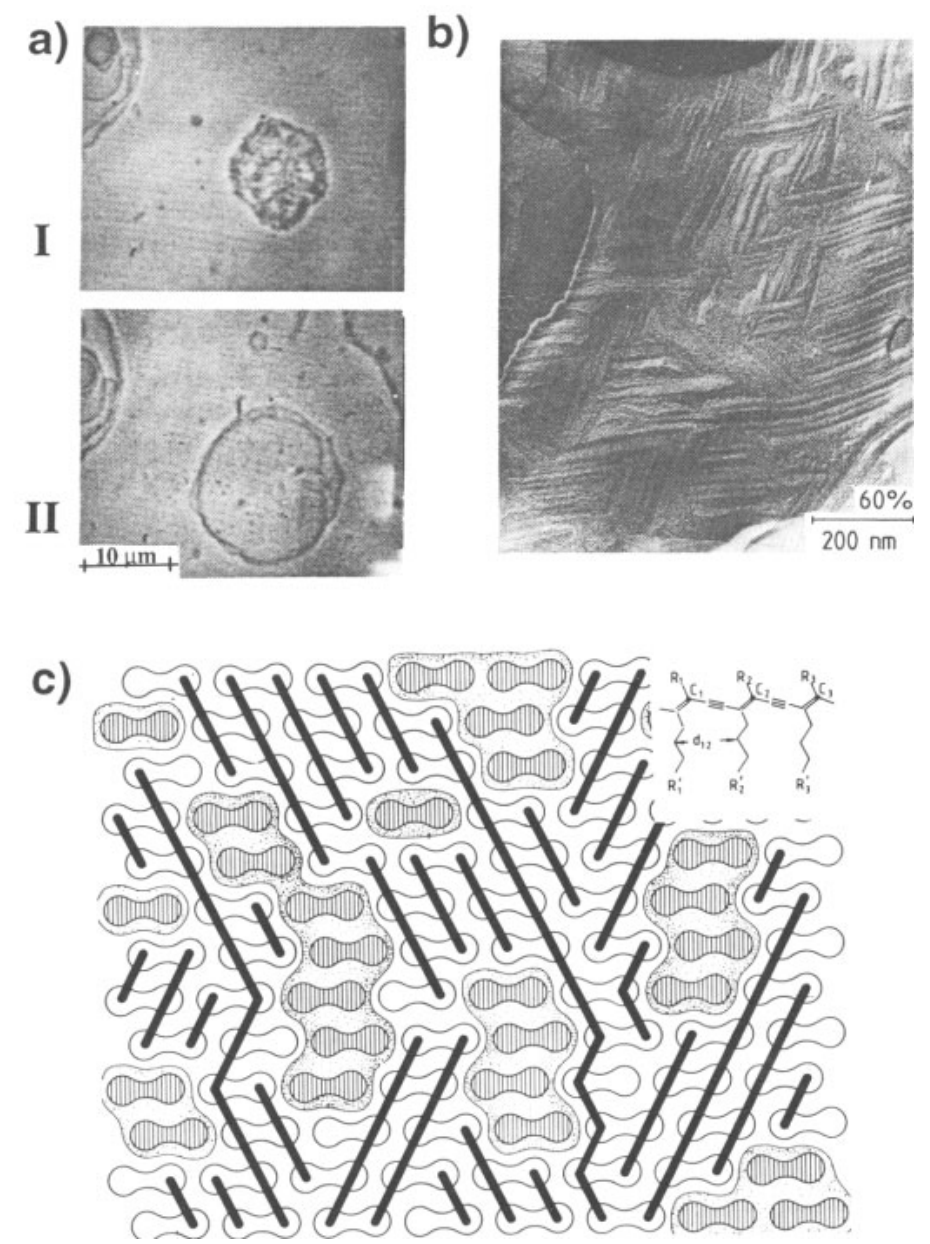

Fig. 10. (a) Wrinkling (or buckling) transition of partially polymerized vesicle from monomeric lecithin and cross-linked diacetylene lecithin above the percolation limit. Phase separation is restricted to microdomain formation. The vesicles still swell above the phase transition of the two lipids (cf. Eggl et al. for details [23]) exhibiting a quasi-spherical shape. They undergo a transition to a strongly wrinkled shape after cooling. The wrinkling transition is reversible. (b) Freeze fracture electron micrograph of weakly wrinkled state. (c) Schematic representation of the polymerized liquid headgroups. 

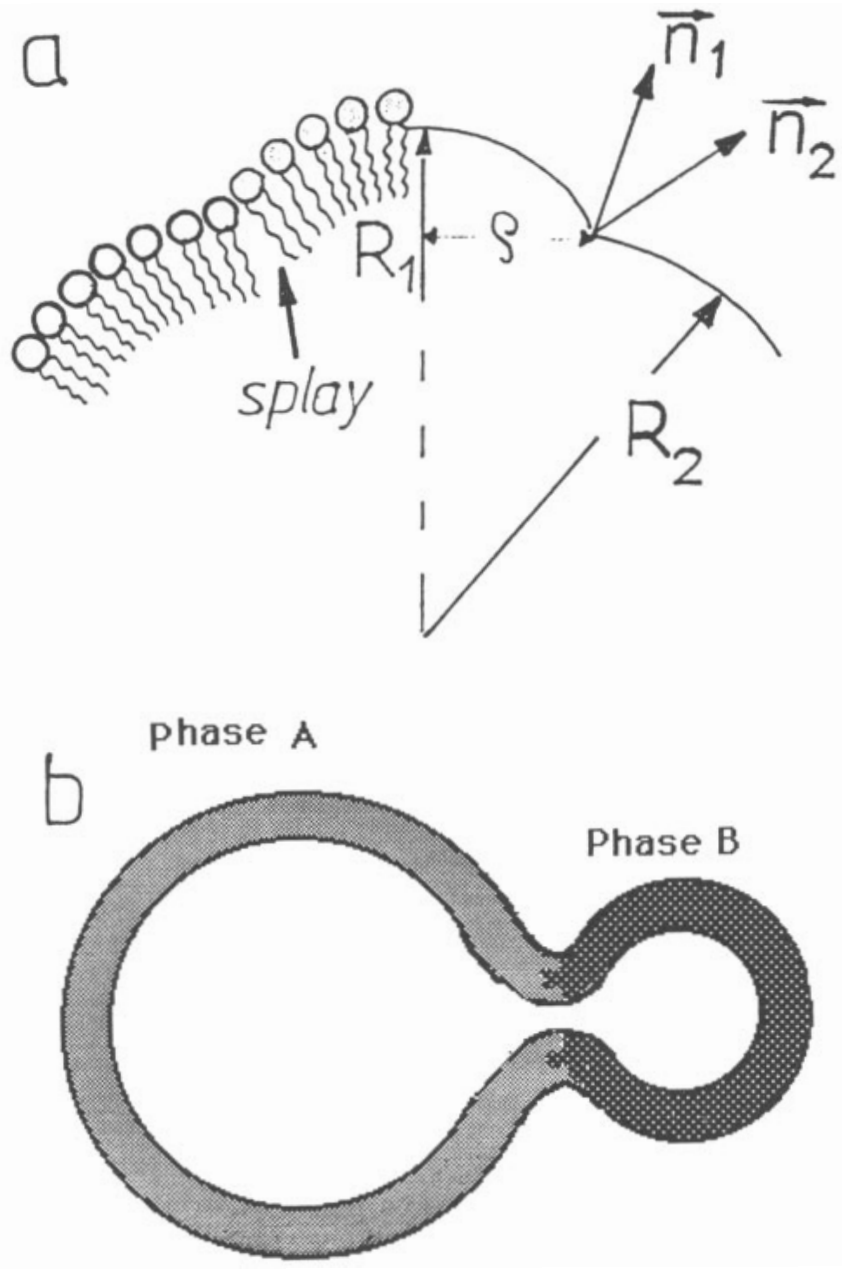

Fig. 11. Two contributions to the interfacial energy of domains formed by lateral phase separation. (a) Splay deformation at interface between regions of different curvature resulting in a positive interfacial energy $\left(\sigma \propto \rho^{2}\right)$ which grows quadratically with the radius $\rho$ of the domains. (b) Reduction of chemical interfacial energy by budding and formation of neck. Note that the neck tends to be unstable.

There are presently two models concerning the stability of vesicles after lateral phase separation and domain formation (cf. Fig. 11).

One is based on the observation that the domains of different composition formed within a bilayer exhibit in general a different curvature (cf. Fig. 11). This leads to an elastic splay deformation at the rims of the domains. It is clear that at constant spontaneous curvature the interfacial energy increases with the square of the domain radius [20]. The splay energy thus counteracts the growth of the $2 \mathrm{D}$ precipitates (e.g. after a spinodal decomposition). It also counteracts the chemical interfacial energy which is expected to favour the growth of the domains until the two phases are completely separated.

Based on this idea a general theory of shape changes of vesicles of lipid alloys has been proposed by combining the Cahn-Hilliard model of spinoidal composition and the spontaneous curvature model $[2,21,24,25]$. The coupling between local curvature and phase separation is considered by including a coupling term in the energy functional. The general Cahn-Hilliard equation has only been solved for the one-dimensional case by Wonneberger [24]. He showed that this equation is isomorphic to the Ginzburg-Landau equation and exhibits sinusoidal concentration profiles as stationary solutions.
Another interesting prediction of this model is that an initial flat membrane becomes globally curved. Leibler and Andelman [25] calculated phase diagrams of stable domain structures as a function of temperature and composition. They found two domains of stable domain structures: (1) stripe-like arrangemens of two-dimensional precipitates and (2) hexagonal arrangements of circular domains. Both types have indeed been observed. Only the second is shown (Fig. 9).

Another possibility is the reduction of the chemical interfacial energy by budding. This situation has recently been treated by Lipowsky [24] who showed that the chemical interfacial energy at a cap formed by phase separation can be reduced by forming a local bud in such a way that the interface resides in the neck interconnecting the bud and the mother vesicle. This results in a new type of budding instability which is driven by the line tension at the rims of the lateral domains formed by lateral phase separation. If the gain in interfacial energy is larger than the bending energy, $W_{\text {bend }}$, associated with the formation of a bud (cf. Fig. 11), budding occurs even without spontaneous curvature of the two-dimensional precipitate.

The radius $R_{\mathrm{b}}$ of the bud may be estimated by minimizing the bending energy and the interfacial energy $\gamma$. To a first approximation

$R_{\mathrm{b}} \sim 8 K_{\mathrm{c}} / \gamma$

In the framework of this model the fission of the budded vesicle could be driven by a negative tension between the bud and the mother vesicle. This tension arises due to bending excitations of the membrane of the mother cell. As is well known these undulations lead to an entropy-driven tension which pulls at the budded vesicle [25] and may lead to the detachment of the bud. Experimental evidence is provided below.

\section{Vesicle fission appears to require lateral phase separation}

Fission requires a destabilization of the neck between the bud and the mother vesicle. It has recently been demonstrated that this instability is driven by lateral phase separation [3]. Thus, if vesicles of natural brain lipid (sphingomyelin) are heated, budding is followed by vesicle fission [23]. Since the lipid consists of a mixture of different chain lengths it exhibits phase separation over a broad temperature regime $\left(30-45^{\circ} \mathrm{C}\right)$. Direct inspection of the process by freeze fracture electron microscopy showed that the lipid composition of the buds differs from that of the mother vesicle.

This process can be explained by the Lipowsky model discussed above. By using eq. (8) one can estimate the interfacial energy. Since at $40^{\circ} \mathrm{C}$ the lipid is in the fluid phase the bending stiffness is $K_{\mathrm{c}} \approx 10^{-12}$ ergs. The radii of the buds are of the order of $1 \mu \mathrm{m}$ and hence $\gamma \sim 10^{-7}$ dynes $/ \mathrm{cm}$.

A biologically important example is shown in Fig. 12. Vesicles composed of a mixture of $60 \%$ DMPC and $40 \%$ cholesterol were osmotically deflated at constant temperature. At increasing the volume, buds form and detach repeatedly. According to small angle neutron scattering studies, cholesterol precipitates within the fluid phase above $40 \mathrm{~mol} . \%$. Since one does not observe large-scale lateral phase separation in this case, the fission mechanism appears 


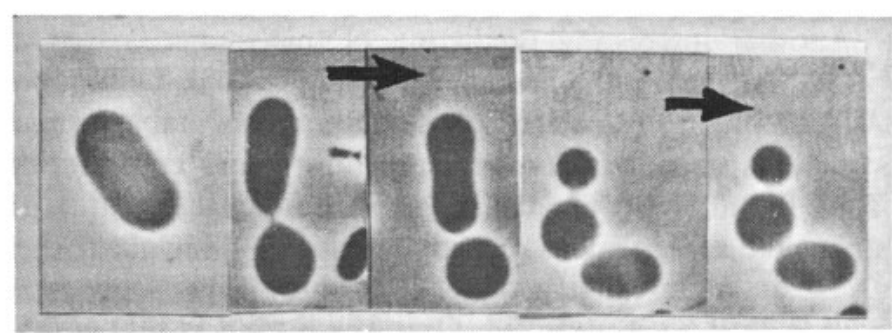

(a)

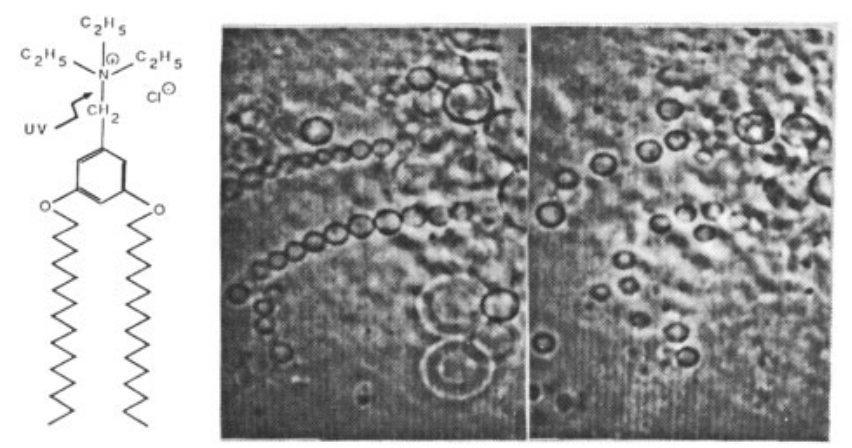

(b)

Fig. 12. Fisson of budded vesicles by local phase separation. (a) Osmotically-driven fission of budded vesicles in binary mixed membranes of DMPC $(60 \mathrm{~mol} . \%)$ and cholesterol $(40 \%)$ after deflation of the vesicle. The separation of the vesicles follows from the fact that they swim apart after fission. Note that biological membranes contain typically $50 \mathrm{~mol} . \%$ cholesterol. (b) Photochemically-induced fission of vesicles caused by local phase separation. The membrane is composed of the amphiphile shown on the left. It can be transformed into a hydrophobic molecule by photochemical cleavage of the hydrophilic ammonium head group. The chain of vesicles explodes into free vesicles within seconds after starting the irradiation.

to be different. It is most likely caused by destabilization of the neck due to local phase separation; that is accumulation of solute in the highly curved region of the neck where the lipid packing density is smaller than in the bulk membrane. The strongly perturbed lipid organisation can be envisaged as a defect. As is well-known, defects in the lipid bilayer provide strongly attractive traps for solutes [2].

More evidence for this explanation is provided by the experiment shown in Fig. 12(b). In this example a chain of vesicles interconnected by necks explodes into free vesicles immediately after photochemical cleavage of some of the amphiphilic molecules. These are transformed into hydrophobic molecules. Since the solubility of these in lipid layers is very low they are expected to form local precipitates. These are again expected to accumulate in the neck where the lipid packing density is low.

The shape changes and global shape instabilities of vesicles of lipid-lipid or lipid-protein mixtures pose fascinating although complex problems. The present studies provide evidence that many complex cellular processes such as phagocytosis, endocytosis or the vesicle budding-fissionfusion sequence of events are governed by simple principles. Many more experimental and theoretical studies are required in order to understand the manifold of shape transitions of vesicles of lipid alloys.

\section{Acknowledgements}

The work was supported by the Deutsche Forschungsgemeinschaft (SFB 266) and the Fonds der Chemischen Industrie. Many stimulating discussions with Rainer Lipowsky and Udo Seifert are gratefully acknowledged.

\section{References}

1. Darnell, J., Lodish, H. and Balitmore, D., "Molecular Cell Biology" (W. H. Freeman, San Francisco 1990).

2. Sackmann, E., Canadian Journal Physics 68, 999 (1990).

3. Bessis, M., "Living Blood Cells and their Ultrastructure" (SpringerVerlag, New York 1973).

4. (a) Zilker, A., Strey, H. and Sackmann, E., in: "The Structure and Conformation of Amphiphilic Membranes" (Springer Proceedings in Physics, Vol. 66) (Edited by R. Lipowsky, D. Richter and K. Kremer) (Springer-Verlag, Berlin, 1992), p. 113. (b) Zilker, A., Ziegler, M. and Sackmann, E., Phys. Rev. A46, 7998 (1992).

5. Zeman, K., Engelhardt, H. and Sackmann, E., Eur. Biophys. J. 18, 203 (1990).

6. Boal, X., Zilker, A. and Seifert, U., Phys. Rev. Lett. 69, 3405 (1992)

7. Berndl, K., Käs, J., Lipowsky, R., Sackmann, E. and Seifert, U., Europhys. Lett. 13, 659 (1990).

8. Käs, J. and Sackmann, E., Biophys. J. 60, 825 (1991).

9. Käs, J., Sackmann, E., Podgorny, R., Svetina, S. and Zeks, B., J. Phys. II France 3, 631 (1993).

10. Bruinsma, R., J. Phys. II France 1, 995 (1991).

11. Bloom, M., Evans, E. and Mouritsen, O., Quart. Rev. Biophys. 24, 293 (1991).

12. (a) Mutz, M. and Bensimon, D., Phys. Rev. A43, 4525 (1991); (b) Mutz, M. and Bensimon, D., Phys. Rev. Lett. 67, 923 (1991).

13. Helfrich, W., Z. f. Naturforsch. 28C, 694 (1973).

14. Evans, E., Biophys. J. 13, 923 (1974).

15. Svetina, S. and Zeks, B., Eur. Biophys. J. 17, 101 (1989).

16. Lipowsky, R., Nature 349, 475 (1991).

17. Seifert, U., Miao, L., Döbereiner, H. G. and Wortis, M., Springer Proceedings in Physics 66, 113 (1992).

18. Sackmann, E., Eggl, P., Fahn, C., Bader, H. and Ringsdorf, H., Ber. Bunsenges. Phys. Chem. 89, 1198 (1985).

19. Petrov, A., Mitov, M. D. and Deizhanski, A. I., in: "Advances in Liquid Crystal Research and Application" (Edited by L. Bataed) (Pergamon Press, Oxford 1980), p. ??.

20. Gebhardt, C., Gruler, H. and Sackmann, E., Z. f. Naturforsch. 32C, 581 (1977).

21. Wonneberger, W., Z. Phys. B46, 73 (1982).

22. Leibler, S. and Andelman, D., J. Phys. France 48, 2013 (1987).

23. Döbereiner, H. G., Käs, J., Noppl., D. and Sackmann, E., Biophys. J. (in press).

24. Lipowsky, R., J. Phys. II. France 2, 1825 (1992).

25. Helfrich, W., Z. f. Naturforsch. 339, 305 (1978). 